\title{
Food introduction and allergy prevention in infants
}

\author{
Elissa M. Abrams MD, Allan B. Becker MD
}

CMAJ Podcasts: author interview at https://soundcloud.com/cmajpodcasts/150364-rev

$\mathrm{A}$ bout $5 \%$ of children and $3 \%-4 \%$ of adults have food allergy. ${ }^{1}$ In a recent Canadian cross-sectional survey that randomly selected households in all 10 Canadian provinces (sampling more than 3000 households), $8.07 \%$ (95\% confidence interval [CI] $7.47 \%-8.67 \%$ ) reported at least one food allergy. ${ }^{2}$ Although almost any food could theoretically cause a reaction, the foods that account for most allergic reactions are cow's milk, soy, wheat, egg, peanut, tree nuts, finned fish, shellfish and sesame. The prevalence of some food allergies has increased, with a reported $18 \%$ increase in overall prevalence between 1997 and 2007 in the United States. ${ }^{3}$ For this reason, the clinical focus on allergy has shifted from treatment to prevention.

Parents often ask how they can prevent food allergy in their infants. Although older guidelines recommended avoidance of the most commonly allergenic foods for at-risk infants in an effort to prevent food allergy, newer guidelines no longer recommend avoidance of such foods beyond four to six months of age.

We discuss current guidelines on food allergy prevention and the evidence behind them, and highlight recent changes in guidance. The evidence we present comes from consensus opinion, systematic reviews and well-conducted randomized controlled trials (Box 1). Most of the recommendations in this review focus specifically on the infant who is at risk for atopy (i.e., an infant with one or more immediate family members with allergic disease).

\section{Why has advice changed?}

In 2000, the American Academy of Pediatrics released a position statement recommending the avoidance of cow's milk until the age of 12 months, eggs until 24 months, and nuts and fish until 36 months in infants at high risk of atopy. ${ }^{4}$ This position was supported by evidence from two studies; one had shown an increased risk of atopic disease with introduction of solid foods at three months compared with six months of age, and the other had shown a correlation between diversity of diet before four months of age and risk of eczema., ${ }^{5,6}$ Overall, the recommendations were based on consensus rather than direct evidence. In addition, the early introduction of foods was hypothesized to increase the risk of allergic disease owing to immune immaturity and gut permeability in infancy. ${ }^{7}$

In 2006, the American College of Allergy, Asthma and Immunology followed suit. ${ }^{8}$ After reviewing 52 studies, the college concluded that early introduction of solid foods could increase the risk of food allergy, and it agreed with the American Academy of Pediatrics' recommendations on delayed introduction of certain foods for children at risk.

A few years after the release of these guidelines, studies began to be published that refuted the notion that delayed introduction was protective. In 2006, a prospective study involving more than 1600 children enrolled at birth and followed until five years of age noted an increased risk of wheat allergy among children exposed to wheat after the age of six months. ${ }^{9}$ In 2008 , a questionnaire study determined that the prevalence of peanut allergy among Jewish schoolchildren in the United Kingdom, where peanut is introduced later, was 10-fold higher than in Israel, where peanut is introduced early and eaten frequently $(p<0.001) .{ }^{10}$ In 2010 , a population-based crosssectional study involving more than 2500 infants noted that early introduction of egg (age four to six months) was associated with a lower prevalence of egg allergy than later introduction
Competing interests: None declared.

This article has been peer reviewed.

Correspondence to:

Elissa Abrams,

elissa.abrams@gmail.com

CMAJ 2015. DOI:10.1503/ cmaj.150364

\section{KEY POINTS}

- Recent evidence has shown that delayed introduction of allergenic foods does not prevent allergic disease.

- Emerging evidence from randomized controlled trials suggests that early introduction of allergenic foods, specifically peanut, is protective against the development of food allergy.

- Although exclusive breastfeeding for six months remains recommended for its many health benefits to both infant and mother, there is no evidence that exclusive breastfeeding or maternal dietary elimination helps prevent food allergy in infants.

- With few exceptions, allergenic foods may be introduced into children's diets between the ages of four and six months; once introduced, regular exposure to the food is important for maintaining tolerance. 
(adjusted odds ratio [OR] 1.6 [95\% CI 1.0-2.6] for introduction at 10-12 months; adjusted OR 3.4 [95\% CI 1.8-6.5] for introduction after 12 months). ${ }^{11}$ In the same year, a prospective study involving more than 13000 infants noted that early exposure to cow's milk (within the first two weeks of life compared with age 105-194 days) was protective against cow's milk allergy $(p<0.001) .{ }^{12}$ Analysis of data from a large prospective birth cohort including more than 2500 infants in the Netherlands showed delayed introduction of foods to be associated with eczema and atopy at the age of two years. ${ }^{13}$ In addition, a retrospective database review including all children born in Manitoba in 1995 found no increased risk of food allergy in premature or low-birthweight children, which refuted the notion that immune system and gastrointestinal tract immaturity increased the risk of food allergy. ${ }^{14}$

In addition to emerging studies that showed that later introduction may be associated with increased risk of allergy, the "dual allergen exposure hypothesis" as an explanation of the causal mechanism of food allergy was proposed by Dr. Gideon Lack. According to his theory, gastrointestinal (GI) exposure (i.e., food consumption) is protective against developing food allergy. The mechanism is thought to relate to the GI tract's function in encouraging immune tolerance. The immune response resulting from cutaneous exposure to an antigen may lead to sensitization, especially in the presence of atopic dermatitis. ${ }^{15}$ Further supporting this hypothesis, a mutation associated with loss of function of the gene encoding filaggrin, a protein involved in skin barrier protection, was discovered. ${ }^{16}$ The mutation is present in about $50 \%$ of patients with eczema. ${ }^{16}$ Filaggrin mutations have also been linked with peanut allergy. ${ }^{17}$

As a result of these new data, the American Academy of Pediatrics released an updated position statement in 2008 that no longer recommended avoidance of any of the allergenic foods beyond the age of four to six months..$^{18}$ A guideline released in 2010 by the National Institute of Allergy and Infectious Diseases supported the

\section{Box 1: Evidence used in this review}

We used Canadian and American national guidelines to inform this review, in addition to published systematic reviews that were known to us. We identified additional articles through MEDLINE literature searches using the search terms "food allergy" and "allergy prevention" from 1990 to present. In addition, we reviewed conference abstracts and reference lists from seminal articles. We restricted our results to English-language articles. Where possible, we selected the most recent articles and the articles with the most robust level of evidence (such as randomized controlled trials and meta-analyses). We reviewed more than 100 citations, of which 38 are included in this review. academy's position, stating that introduction of solid food need not be delayed beyond four to six months of age..$^{19}$ The American Academy of Allergy, Asthma and Immunology also noted that studies were emerging that may support early introduction as being protective against food allergy. ${ }^{20}$ In 2013, the Canadian Pediatric Society, in conjunction with the Canadian Society of Allergy and Clinical Immunology, released a new guideline on dietary exposures and allergy prevention, which noted that delayed introduction of allergenic foods was not thought to be protective against the development of allergic disease. ${ }^{21}$ The guideline states that although at the time of its release the benefits of early introduction were "yet to be determined," "immunological mechanisms of sensitization and tolerance tend to support [early introduction]." The guideline discouraged routine preemptive testing before food introduction and suggested referral to an allergist if families were reluctant to introduce a food.

\section{Are there exceptions to the new guidance?}

One exception to the advice to no longer delay introducing allergenic foods is for infants with older siblings with peanut allergy, because an almost sevenfold increased risk in the younger sibling (OR 6.72 [95\% CI 2.04-22.12]) has been documented. In such circumstances, evaluation by a certified allergist before peanut introduction in the younger sibling is recommended. ${ }^{22}$ Any infant with a documented reaction to a food should undergo assessment by an allergist before the food is introduced again. In addition, American Academy of Allergy, Asthma and Immunology recommends that infants with moderate to severe atopic dermatitis be evaluated by a specialist before allergenic foods are introduced. ${ }^{20}$

\section{Could early food introduction prevent development of allergy?}

Evidence from randomized trials is now available to help us determine whether early introduction of allergenic foods could prevent allergy development, specifically with regard to peanut allergy. The recent Learning Early About Peanut (LEAP) study is the first prospective, randomized controlled trial to show a relative risk reduction of up to $80 \%$ with early — versus late peanut introduction in children at high risk for allergy. ${ }^{23}$ The trial randomly assigned 640 infants at high risk for peanut allergy (due to 
atopic dermatitis, egg allergy or both) to either regular peanut consumption (three or more servings per week) or avoidance until the age of five years. The primary outcome was rate of peanut allergy at the age of five years. Children were excluded if a baseline skin prick test to peanut had a wheal with a diameter of more than $4 \mathrm{~mm}$.

In the group that ate peanut regularly, the overall rate of peanut allergy was $3.2 \%$ compared with $17.2 \%$ in the avoidance group $(p<0.001)$. Among children who had an initial negative skin test result to peanut, the rate of allergy was $1.9 \%$ in the consumption group, compared with $13.7 \%$ in the avoidance group ( $86.1 \%$ relative reduction). For children who initially had a positive skin test result to peanut, the rate of peanut allergy was $10.6 \%$ in the consumption group and $35.3 \%$ in the avoidance group (70\% reduction in prevalence).

The authors of an editorial that accompanied publication of the trial recommended that any child between the ages of four and eight months at high risk for peanut allergy should undergo skin prick testing. ${ }^{24}$ If the result is negative, peanut should be introduced into the diet on a regular basis. If the result is positive, evaluation by an allergist should help to determine future management, which may include an oral challenge if the skin test showed mild sensitivity (wheal $\leq 4 \mathrm{~mm}$ ).

As a result of the findings of the LEAP study, a new consensus communication on behalf of the American Academy of Allergy, Asthma and Immunology, American Academy of Pediatrics and Canadian Society of Allergy and Clinical Immunology (among other organizations) has been released that states that for infants at high risk (as defined by the LEAP trial), strong evidence exists for peanut introduction early in life (between ages of 4 and 11 months). ${ }^{25}$ The communication also states that these infants may first benefit from evaluation by an allergist before peanut introduction. More formal guidelines are anticipated from the National Institute of Allergy and Infectious Diseases.

Questions remain as to whether early food introduction prevents allergy development (Box 2). One such consideration is whether the results of the LEAP study may be applicable to other highly allergenic foods. The ongoing Enquiring About Tolerance (EAT) study seeks to answer that question ${ }^{26}$ by examining early introduction (at three months of age) of peanut, sesame, wheat, fish, egg and cow's milk compared with standard introduction (age six months or older). The main outcome measure is clinical allergy at three years of age. The Hens Egg Allergy Prevention (HEAP) study (German Clinical Trials Register No. DRKS-ID:
DRKS00005668) is examining whether early introduction of egg (three times a week starting at four months of age) is protective compared with more delayed introduction (evaluation at one year). Another consideration is whether early introduction of peanut will provide long-lasting protection against peanut allergy, which is being investigated by the ongoing LEAP (LEAP-On) trial. Whether early peanut introduction will be protective in children at lower risk for allergy is not known. Finally, the absolute amount of peanut that needs to be consumed and the frequency of peanut exposure required to be protective against peanut allergy is still to be determined.

\section{What roles do maternal avoidance diets and breastfeeding play?}

Current guidelines do not support maternal avoidance diets during pregnancy or lactation. ${ }^{18,19}$ A systematic review published in 2010 concluded that the evidence was not clear that maternal exposure to peanut had any subsequent effect on the development of peanut allergy in the child. ${ }^{27}$

A Cochrane review concluded that maternal avoidance diets in pregnancy were unlikely to reduce risk of atopic disease in childhood. In addition, the review noted that allergen avoidance had been shown in some studies to adversely affect maternal or fetal nutrition, leading to lower mean birth weight, higher risk of preterm birth and lower gestational weight gain. ${ }^{28}$ Maternal avoidance diets during lactation did not reduce risk of atopic disease in children, with the possible exception of atopic dermatitis, although this was largely based on a single trial. The Cochrane review concluded that "better trials are needed."

Exclusive breastfeeding for six months is recommended by the World Health Organization, American Academy of Pediatrics and Canadian Pediatric Society for its many health benefits for both mother and child. ${ }^{29-31}$ However, whether breastfeeding is protective against allergic disease is unclear. Although a systematic review and meta-analysis in 2001 noted that exclusive

\section{Box 2: Unanswered questions}

- Is early introduction of foods other than peanut also protective against food allergy?

- Once introduced, what is the frequency and amount of food that needs to be eaten to ensure maintenance of tolerance?

- Does early introduction provide only shortterm protection against food allergy, or is the protection long-lasting? 
breastfeeding for the first three months of life lowered rates of eczema during childhood (in children with an immediate family history of allergy only), ${ }^{32}$ other studies have not replicated this protective effect. ${ }^{33,34}$ Exclusive breastfeeding decreases early childhood viral wheeze, but it has not been shown to protect against long-term asthma risk. ${ }^{35,36}$ In fact, some studies show an increased long-term risk of asthma with exclusive breastfeeding. ${ }^{37}$ The PROBIT (Promotion of Breastfeeding Intervention Trial) trial, a cluster randomized trial including more than 17000 mother-infant pairs, noted no protective effect of prolonged and exclusive breastfeeding on asthma or aeroallergen sensitization..$^{38}$ The Canadian Pediatric Society guideline on dietary exposures concludes that it is not yet known whether breastfeeding protects against allergy. ${ }^{21}$

\section{How should we counsel families on food introduction?}

The recent statement from the American Academy of Allergy, Asthma and Immunology provides practical advice for families around complementary food introduction. ${ }^{20}$ It recommends that new foods be introduced at a rate of no more than one new food every three to five days in an age-appropriate manner (to avoid choking risk). In addition, the statement suggests starting with typical complementary foods (grains, yellow or orange vegetables, fruit) and introducing one of the highly allergenic foods once a few complementary foods have been well tolerated. The highly allergenic foods should be given at home (i.e., not at daycare), and the guideline reiterates that reactions can occur with first known ingestion. If there is no reaction, it is recommended that the food in question be gradually increased in amount over a few days' duration. As stated in the Canadian Pediatric Society's guideline, regular ingestion of tolerated foods is important for maintenance of tolerance. ${ }^{21}$ Once a highly allergenic food is tolerated, it should be consumed regularly (although the optimal frequency is not known).

\section{Conclusion}

At this stage, it has been well documented that avoidance of allergenic foods is not preventive of food allergy. In the newly released LEAP study, there is strong evidence that early introduction of peanut is in fact preventive. How this will change current guidelines on food introduction remains to be seen.

If a family asks how to prevent allergy in their children, our current advice is to introduce the allergenic foods at four to six months of age. Once highly allergenic foods are introduced, regular exposure is important for maintenance of tolerance — children should eat these foods on a regular basis. Although breastfeeding should be recommended for its many positive benefits, we cannot advise breastfeeding or maternal dietary elimination as methods of allergy prevention. Any child who has a sibling with peanut allergy, who has a history of an immediate reaction to a food or who has moderate to severe atopic dermatitis should undergo assessment by an allergist. The evaluating allergist should prioritize the evaluation of infants at risk in an effort to avoid prolonged wait lists, which could result in missing the window of opportunity for early food introduction.

\section{References}

1. Sicherer SH, Sampson HA. Food allergy. J Allergy Clin Immunol 2010;125:S116-25.

2. Soller L, Ben-Shoshan M, Harrington DW, et al. Overall prevalence of self-reported food allergy in Canada. J Allergy Clin Immunol 2012;130:986-8.

3. Branum AM, Lukacs SL. Food allergy among children in the United States. Pediatrics 2009;124:1549-55.

4. American Academy of Pediatrics, Committee on Nutrition. Hypoallergenic infant formulas. Pediatrics 2000;106:346-9.

5. Kajosaari M. Atopy prevention in childhood: the role of diet Prospective 5-year follow up of high-risk infants with six months exclusive breastfeeding and solid food elimination. Pediatr Allergy Immunol 1994;5(Suppl):26-8.

6. Fergusson DM, Horwood LJ, Shannon FT. Early solid feeding and recurrent childhood eczema: a 10-year longitudinal study. Pediatrics 1990;86:541-6.

7. Høst A, Koletzko B, Dreborg S, et al. Dietary products used in infants for treatment and prevention of food allergy. Joint statement of the European Society for Paediatric Allergology and Clinical Immunology (ESPACI) Committee on Hypoallergenic Formulas and the European Society for Paediatric Gastroenterology, Hepatology, and Nutrition (ESPGHAN) Committee on Nutrition. Arch Dis Child 1999;81:80-84.

8. Fiocchi A, Assa'ad A, Bahna S. Adverse reactions to food committee: American Academy of Allergy, Asthma and Immunology. Food allergy and the introduction of solid foods to infants: a consensus document. Ann Allergy Asthma Immunol 2006;97:10-20.

9. Poole JA, Barriga K, Leung DY et al. Timing of initial exposure to cereal grains and the risk of wheat allergy. Pediatrics 2006; 117:2175-82.

10. Du Toit G, Katz Y, Sasieni P, et al. Early consumption of peanuts in infancy is associated with a low prevalence of peanut allergy. J Allergy Clin Immunol 2008;122:984-91.

11. Koplin JJ, Osborne NJ, Wake M, et al. Can early introduction of egg prevent egg allergy in infants? A population-based study. $J$ Allergy Clin Immunol 2010;126:807-13.

12. Katz Y, Rajuan N, Goldberg MR, et al. Early exposure to cow's milk is protective against IgE-mediated cow's milk protein allergy. J Allergy Clin Immunol 2010;126:82.e1.

13. Snijders BE, Thijs C, van Ree R, et al. Age at first introduction of cows' milk products and other food products in relation to infant atopic manifestations in the first 2 years of life: the KOALA birth cohort study. Pediatrics 2008;122:e115-22.

14. Liem JJ, Kozyrskyj AL, Huq SL, et al. The risk of developing food allergy in premature or low-birth-weight children. J Allergy Clin Immunol 2007;119:1203-9.

15. Lack G. Uptdate on risk factors for food allergy. J Allergy Clin Immunol 2012;129:1187-97.

16. O'Regan GM, Irvine AD. The role of filaggrin in the atopic diathesis. Clin Exp Allergy 2010;40:965-72.

17. Asai Y, Greenwood C, Hull PR, et al. Filaggrin gene mutation associations with peanut allergy persist despite variations in peanut allergy diagnostic criteria or asthma status. J Allergy Clin Immunol 2013;132:239-42.

18. Greer FR, Sicherer SH, Burks AW; American Academy of Pediatrics Committee on Nutrition. American Academy of Pediatrics Section on Allergy and Immunology. Effects of early nutritional 
interventions on the development of atopic disease in infants and children: the role of maternal dietary restriction, breastfeeding, timing of introduction of complementary foods, and hydrolyzed formulas. Pediatrics 2008;121:183-91.

19. Boyce JA, Assa'ad A, Burks AW, et al. Guidelines for the diagnosis and management of food allergy in the United States: report of the NIAID-sponsored expert panel. J Allergy Clin Immunol 2010;126(Suppl):51-8.

20. Fleischer DM, Spergel J, Assa'ad A. Primary prevention of allergic disease through nutritional interventions. J Allergy Clin Immunol Practice 2013;1:29-36.

21. Chan ES, Cummings C. Dietary exposures and allergy prevention in high-risk infants. Paediatr Child Health 2013;18:545-54

22. Liem JJ, Huq S, Kozyrskyj Al et al. Should younger siblings of peanut-allergic children be assessed by an allergist before being fed peanut? Allergy Asthma Clin Immunol 208;4(4):144-9.

23. Du Toit G, Roberts G, Sayre P, et al. Randomized trial of peanut consumption in infants at risk for peanut allergy. N Engl J Med 2015;372:803-13.

24. Gruchalla RS, Sampson HA. Preventing peanut allergy through early consumption — ready for prime time? N Engl J Med 2015;372:875-7.

25. Consensus communication on early peanut introduction. Milwaukee (WI): World Allergy Organization. Available: www.worldallergy.org/consensus-communication-early-peanut -introduction (accessed 2015 June 15)

26. EAT study [homepage]. London (UK): Enquiring About Tolerance. Available: www.eatstudy.co.uk/eat-study-info/ (accessed 2015 Mar. 1).

27. Thompson RL, Miles LM, Lunn J, et al. Peanut sensitisation and allergy: influence of early life exposure to peanuts. Br J Nutr 2010;103:1278-86.

28. Kramer MS, Kakuma R. Maternal dietary antigen avoidance during pregnancy or lactation, or voth, for preventing or treating atopic disease in the child. Evid Based Child Health 2014;9:447-83.

29 Fifty-fifth World Health Assembly on Infant and Child Nutrition. Geneva: World Health Organization; 2002.

30. Section on Breastfeeding. Breastfeeding and the use of human milk. Pediatrics 2012;129:e827-41.

31. Critch JN. Nutrition for healthy term infants, birth to six months: an overview. Paediatr Child Health 2013;18:206-9.

32. Gdalevich M, Mimouni D, David M, et al. Breast-feeding and the onset of atopic dermatitis in childhood: a systematic review and meta-analysis of prospective studies. J Am Acad Dermatol 2001;45:520-7.

33. Bergmann RL, Diepgen TL, Kuss O, et al. Breastfeeding duration is a risk factor for atopic dermatitis. Clin Exp Allergy 2002;32:205-9.
34. Pesonen M, Kallio MJ, Ranki A, et al. Prolonged exclusive breastfeeding is associated with increased atopic dermatitis: a prospective follow-up study of unselected health newborns from birth to age 20 years. Clin Exp Allergy 2006;36:1011-8.

35. Giwercman C, Halkjaer LB, Jensen SM, et al. Increased risk of eczema but reduced risk of early wheezy disorder from exclusive breast-feeding in high-risk infants. J Allergy Clin Immunol 2010;125:866-71.

36. Kull I, Almqvist C, Lilja G, et al. Breastfeeding reduces the risk of asthma during the first 4 years of life. J Allergy Clin Immunol 2004; 114:755-60.

37. Sears MR, Greene JM, Willan AR, et al. Long-term relation between breastfeeding and development of atopy and asthma in children and young adults: a longitudinal study. Lancet 2002;360:901-7.

38. Kramer MS, Matush L, Vanilovich I, et al. Effect of prolonged and exclusive breast feeding on risk of allergy and asthma: cluster randomized trial. BMJ 2007;335:815.

Affiliations: Pediatric Allergy and Clinical Immunology, University of Manitoba, Winnipeg, Man.

Contributors: Both of the authors wrote the article and revised it for important intellectual content. Both of the authors approved the version to be published and agree to act as guarantors of the work.

\section{Resources for physicians}

- Canadian Pediatric Society statement on food introduction: Chan E, Cummings C. Dietary exposures and allergy prevention in highrisk infants. Pediatr Child Health 2013;18:545-54.

- Consensus communication on early peanut introduction and the prevention of peanut allergy in high-risk infants: www.worldallergy.org/ consensus-communication-early-peanut-introduction (accessed 2015 June 15).

- American Academy of Allergy, Asthma and Immunology statement on food introduction: Fleischer D, Spergel J, Assa'ad A. Primary prevention of allergic disease through nutritional interventions. J Allergy Clin Immunol: In Practice 2013;1:29-36.

- Learning Early About Peanut (LEAP) study: Du Toit G, Roberts G, Sayre P, et al. Randomized trial of peanut consumption in infants at risk for peanut allergy. NEJM 2015;372:803-13. 